\title{
Resources, Fertility, and Parental Investment in Mao's China
}

\author{
Tih-Fen Ting
}

University of Michigan

\begin{abstract}
During the first three decades of the People's Republic of China, income differences across social classes were compressed. Formal education was not an important determinant of personal income. In this study, there was no difference in income between white-collar and blue-collar families, although white-collar parents had more education than blue-collar parents. In urban China, where population density was high, couples of different occupational status appeared to make different trade-offs between quantity and quality of children. Urban white-collar couples had fewer, but better-educated, children than their blue-collar counterparts. In rural areas, white-collar couples still had better educated children than blue-collar couples, but no difference was found in lifetime reproductive success. High population density and an occupational structure that incidentally helped reinforce unequal distribution of cultural capital in the population encouraged urban couples with different levels of resources (i.e., cultural capital rather than income in this case) to adopt different reproductive strategies.
\end{abstract}

KEY WORDS: parental investment; fertility; cultural capital; education; occupation; China.

\section{INTRODUCTION}

All organisms with finite resources face two fundamental trade-offs when it comes to reproduction (Roff, 1992; Stearns, 1992). First, they must "decide" how many resources to expend on reproduction instead of on self-growth and maintenance. Second, they must determine how to divide

Please address correspondence to Tih-Fen Ting, Department of Environmental Studies, University of Illinois at Springfield, Springfield, IL 62703; e-mail: tting1@uis.edu 
the resources available for reproduction between the quantity and the quality of their offspring (Clutton-Brock \& Godfray, 1991; Lessells, 1991).

In this paper, I explore how and why married couples with different occupational status in different parts of China adopt different reproductive strategies. Successful reproductive strategies depend in part on the intensity of competition. In other species, the density of con-specific competitors in the environment is a reflection of this competition (MacArthur \& Wilson, 1967). For individuals living in densely populated (or otherwise highly competitive) environments, the most successful reproductive strategy is often not to make the maximum number of offspring, but to make fewer, better-invested ones (Low, 2000a, 2000b; MacArthur \& Wilson, 1967).

Humans, of course, are more complex and variable than other species, so we need additional measures. Still, human parental investment strategies (like those of other species) are sensitive to the amount, distribution, and accessibility of environmental resources (Lancaster, 1997; Low, 2000b). When the marginal rates of return on investment in children's ability to survive, marry, and reproduce are high, we expect that parents would respond to those investment opportunities, even at the expense of the number of children (Charnov, 1982; Becker, 1991; Low, Clarke, \& Lockridge, 1992).

Parental investment comes in many different forms. In modern societies, education is important because it is crucial for social mobility. Scholars of education and social stratification suggest that, in all industrialized or industrializing societies for which data are available, people believe those who get ahead are those who get educated (see Deng \& Treiman, 1997). Thus, education cost constitutes a significant part of parental investment, at least in modern societies.

With differentials in resource availability and opportunities for better-educated children, we expect that married couples would adopt different parental investment strategies in different environments. For example, couples of relatively high occupational status (e.g., white-collar) might opt for offspring quality over offspring quantity in urban settings where population density is high and the skilled labor market is competitive. On the other hand, couples of lower occupational status (e.g., blue-collar) might invest in offspring quantity instead of quality, if relatively limited resources make it harder for them to invest more per child. In rural areas where population density is low and the labor market is mainly based on unskilled labor, we expect to see a lesser degree of differentiation in parental investment strategies among couples of different occupational status (cf. Goody, 1983).

China is an interesting case in which to study parental investment strategies for several reasons. During the first three decades of the People's Republic of China, people experienced dramatic social, cultural, and 
TIH-FEN TING

political transformation as a result of various government policies (Meisner, 1999; Schoppa, 2002; Ting, 2003, this issue). Many of these policies specifically aimed to equalize people's access to material resources and educational opportunities by eliminating social stratification. These policies included land reform, agricultural collectivization in rural areas, and nationalization of urban industry (Schoppa, 2002). As a result, in rural areas private ownership of land (and of all other forms of wealth except housing) was abolished, and material resources became the common property of the newly created communes and brigades (Stockman, 2000). The class structure of urban society was also transformed; private businesses were collectivized. The whole urban population was then assigned to "work-units" by the communist government, through which welfare and social security were provided (Stockman, 2000).

However, not only material resources are useful investments in children: "cultural capital" also is important. In this study, the term has a broader meaning than is usually recognized. That is, not only are parents able to pass down the knowledge to their offspring because of their educational qualification and professional expertise (i.e., cultural capital in a stricter sense), but also provide resources such as high-quality schooling and collective goods associated with their work-units. Therefore, even though education per se does not have the conventional exchange value for higher income (or wealth), education leads to different professions of different work-units, which in turn gives rise to differentials in fringe benefits. Moreover, one might expect that people with different cultural capitals also tend to be active in different circles (i.e., different networks), which at times might be conducive to personal advancement through private but yet selective association (see also Stockman, 2000).

Communist policies went some way toward interrupting the inheritance of cultural capital down the generations, with an aim of reducing inequalities of educational opportunity among social classes (Stockman, 2000). In particular, from the beginning of the communist regime in 1949, through the Cultural Revolution which ended in 1976, the Chinese government promoted educational opportunities for the children of peasants and workers, favoring them at the expense of those from higher status backgrounds (see Deng \& Treiman, 1997).

Despite the government's efforts to reduce material inequalities, regions varied enormously in their natural endowments and levels of economic development. Egalitarianism of the commune system was not efficient in redressing this resource differential. In fact, the state policy of local self-reliance reinforced interregional inequalities, and the gap of school provision between cities and countryside remained wide (Stockman, 2000). 
POPULATION AND ENVIRONMENT

State interventions for reducing class differences during the first three decades of the People's Republic of China obviously increased the resources of some couples and decreased others. Did couples of different occupational backgrounds also converge on their parental investment strategies? Or did white-collar couples still choose offspring quality over quantity (and vice versa for blue-collar couples)? Was there any difference in the parental investment strategies between urban and rural couples or couples from different regions of China?

To answer these questions, I compare the lifetime reproductive success, and years of education children received, for married couples of different occupational status from different parts of China (Hebei, Shaanxi, and Shanghai). Specifically, I focus on women who were at the end of their reproductive careers in the mid-1980s: their children were born and grew up during the first three decades of the communist regime when various policies for eliminating inequalities took place.

To incorporate the effects of region, I examine women's lives in three provinces (although Shanghai is actually a municipality). Hebei, Shaanxi, and Shanghai have different ecological, demographic and economic characteristics. Shanghai includes the biggest city in China, and is the most economically developed site. Hebei and Shaanxi are rural. Shaanxi is the poorest of the three provinces. Comparisons across provinces reveal some differences in women's reproductive strategies; however, there are many co-variates across whole provinces. Thus, within-province comparisons are required to examine in detail how regional variations or inequalities affect the life history strategies for people of different backgrounds in these different environments.

\section{MATERIALS AND METHODS}

Hebei, Shaanxi, and Shanghai have different ecological, demographic, and economic characteristics. Hebei, a coastal and mainly rural province, is located on the North China Plain. Shaanxi, an inland rural province on the middle reaches of the Yellow River, is located in the center of China. Situated just south of the Yangtze Estuary, Shanghai is one of the largest ports in China and biggest cities in the world. Although, it is mainly urban, Shanghai still has a sizeable (about 38\%) rural population (Dankert \& van Ginneken, 1991). Moreover, Shanghai consistently has the highest population density in China, while Shaanxi has the lowest among the three study areas (Wang, 2000). For more details regarding these three areas, see Ting (2003, this issue). 


\section{Data}

I use data from the 1985 China In-depth Fertility Survey (IDFS), phase I, carried out by the Department of Population Statistics of the State Statistical Bureau (SSB) of the PRC, in cooperation with the International Statistical Institute, Voorburg, The Netherlands. The IDFS was largely inspired by the example of the World Fertility Survey carried out between 1973 and 1985. Details of survey methodology and main findings were published in the Principle Report (SSB, 1986; see also Ting, this issue).

Although, the survey covered women aged 15-49 years, here I consider only those who were aged 45-49 and still in their first marriage. I chose women aged 45-49 because they had completed their reproductive career, and data on their lifetime reproductive success were available. I focus on women who were still in their first marriage to control for possible differential treatment of children of different paternity (Anderson et al., 1999). Despite the first marriage criterion, I consider this selected sample of women to be representative, because the majority of women aged 45-49 when the survey took place were still in their first marriage. Over $90 \%$ of women in Hebei and Shanghai, and $85 \%$ of women in Shaanxi, were still in their first marriage when the survey took place. This sample includes 524 women from Hebei, 429 from Shaanxi, and 464 from Shanghai.

\section{Occupational Classification}

I classify different family backgrounds solely based on husbands' occupations, because $45 \%$ of women in the selected sample said they never worked (more likely they meant they worked without compensation). I group husbands' occupations, which make up approximately 150 occupational codes, into two different categories: white-collar professions and blue-collar professions.

White-collar professions include professionals and administrators (cf. Lin \& Xie, 1988). Professionals are scientific researchers, physicians, economic planners, legal professionals, engineering technicians, teachers, health technicians and assistants, finance and accounting staff, and so forth. Administrators are managers for government agencies, party committees, people's organizations and institutions. Blue-collar jobs include clerical/sales workers, service workers, peasants and manual workers (cf. Lin \& Xie, 1988). Clerical/sales workers include administrative staff, salesmen, procurement staff, and so on. Service workers are various attendants, cooks and kitchen staff, and repairmen for household goods. Peasants are mostly grain farmers. Manual workers include miners, foundry workers, 
POPULATION AND ENVIRONMENT

chemical workers, textile workers, food-processing workers, bench workers, machine operators, electricians, construction workers, and so forth.

Analysis

Income. When considering trade-offs, "phenotypic correlation" often obscures the issue at hand (Lessells, 1991; Low, 2000a, pp. 16-17). A tradeoff occurs when a limiting resource can be spent on one thing or the other, but not both. However, individuals often differ in their resources, and they may also differ in how they might best allocate their resources (Lessells, 1991). Thus, comparing individuals with different levels of income might not reveal a trade-off. For example, if couples with different occupations also differ in their income, those with more money can choose to invest more and have more children at the same time, rather than focus only on offspring quality. As a result of this "phenotypic correlation" we might not observe the trade-off between the quantity and quality of offspring. Therefore, I test whether there are differences in incomes among couples of different occupation.

Lifetime reproductive success. I use multiple regression analysis to examine how married couples from each of the study areas (Hebei, Shaanxi, and Shanghai) with different occupational backgrounds differed in their lifetime reproductive success. For each province, I use separate analyses for all couples, only urban couples, and only rural couples.

I use survey information on the number of living children (not children ever-born) as an estimate of a couple's lifetime reproductive success. Husband's occupation is an independent variable in all analyses.

Education. I use multiple regression to test for differences in the number of years of education received for children born to couples of different occupational status in each of the three provinces. For each province, three analyses were conducted. The first applied to all children; the second included only urban children and the third only rural children.

I calculate the numbers of years of education children have based on the survey information on their education levels (primary, secondary, senior high, and college) and their grade at that particular level. Only children aged seven and older are included in the analyses, because seven is the typical age for starting elementary school (Hannum \& Xie, 1994). I control for both child's age and sex in all analyses for the following reasons. First, the older the child is, the more schooling she/he is likely to have. Second, historically there has been a gender gap in educational attainment in China; sons are usually favored over daughters (Lavely, Xiao, Li, \& Freedman, 1990). 
TIH-FEN TING

\section{RESULTS}

\section{Income}

"Income" refers to a couple's present monthly income when the survey was conducted in 1985. Across provinces, urban couples had higher monthly income than rural couples; however, white-collar families did not have higher income than blue-collar families, whether in urban or rural parts of each province (Tables 1-a to 1-c). With the exception of the urban-rural divide, the income distribution tended to be egalitarian among families of different backgrounds. Thus, it is appropriate to compare white-collar with blue-collar workers regarding their investment allocation among children in this case.

\section{Lifetime Reproductive Success}

When examining the province as a whole, white-collar couples had fewer living children than blue-collar couples in Hebei and Shanghai. Urban couples also had fewer living children than rural couples in Shaanxi and Shanghai (Tables 2a-c). Within each province, white-collar couples in urban areas had fewer living children than their blue-collar counterparts. However, among rural couples, there was no difference in lifetime reproductive success between white-collar and blue-collar workers (Tables $2 \mathrm{a}-\mathrm{c}$ ).

\section{Table 1a}

Multiple Regression Analysis on Monthly Income According to Husband's Occupation: Hebei Province

\begin{tabular}{|c|c|c|c|c|c|c|}
\hline \multirow[b]{2}{*}{ Intercept } & \multicolumn{2}{|c|}{ Total $^{\mathrm{a}}$} & \multicolumn{2}{|c|}{$U_{\text {rban }}^{b}$} & \multicolumn{2}{|c|}{ Rural $^{\mathrm{C}}$} \\
\hline & $\begin{array}{c}\text { Coefficient } \\
74.79\end{array}$ & $\begin{array}{l}\text { S.E. } \\
3.82^{* * * *}\end{array}$ & $\begin{array}{c}\text { Coefficient } \\
134.54\end{array}$ & $\begin{array}{c}\text { S.E. } \\
8.56^{* * * *}\end{array}$ & $\begin{array}{c}\text { Coefficient } \\
73.76\end{array}$ & $\begin{array}{c}\text { S.E. } \\
3.63^{* * * *}\end{array}$ \\
\hline $\begin{array}{l}\text { Urban versus Rural } \\
\text { White-versus } \\
\text { Blue-collar }^{\mathrm{e}}\end{array}$ & $\begin{array}{r}56.15 \\
7.52\end{array}$ & $\begin{array}{l}7.39^{* * * *} \\
9.09\end{array}$ & -5.23 & 16.10 & 17.47 & 11.29 \\
\hline
\end{tabular}

${ }^{\mathrm{a}} \mathrm{N}=524, \mathrm{~F}(2,521)=32.19, p<0.0005, R^{2}=0.11$.

${ }^{\mathrm{b}} \mathrm{N}=138, \mathrm{~F}(1,136)=0.11, p=0.75, R^{2}=0.00$.

${ }^{\mathrm{c}} \mathrm{N}=386, \mathrm{~F}(1,384)=2.40, p=0.12, R^{2}=0.01$.

${ }^{\mathrm{d}}$ Residence: urban $=1$, rural $=0$

e Husband's occupation: white-collar $=1$, blue-collar $=0$.

$* * * * p<0.0005$. 
Table 1b

Multiple Regression Analysis on Monthly Income According to Husband's Occupation: Shaanxi Province

\begin{tabular}{|c|c|c|c|c|c|c|}
\hline \multirow[b]{2}{*}{ Intercept } & \multicolumn{2}{|c|}{ Total $^{\mathrm{a}}$} & \multicolumn{2}{|c|}{ Urban $^{\mathrm{b}}$} & \multicolumn{2}{|c|}{ Rural $^{\mathrm{C}}$} \\
\hline & $\begin{array}{c}\text { Coefficient } \\
52.39\end{array}$ & $\begin{array}{l}\text { S.E. } \\
3.58^{* * * *}\end{array}$ & $\begin{array}{c}\text { Coefficient } \\
124.25\end{array}$ & $\begin{array}{c}\text { S.E. } \\
8.67^{* * * *}\end{array}$ & $\begin{array}{c}\text { Coefficient } \\
52.67\end{array}$ & $\begin{array}{c}\text { S.E. } \\
3.01^{* * * *}\end{array}$ \\
\hline $\begin{array}{l}\text { Urban versus Rural } \\
\text { White-versus } \\
\text { Blue-collar }^{\mathrm{e}}\end{array}$ & $\begin{array}{l}72.77 \\
11.38\end{array}$ & $\begin{array}{c}6.76^{* * * *} \\
8.73\end{array}$ & 14.28 & 15.50 & 7.63 & 10.87 \\
\hline
\end{tabular}

${ }^{\mathrm{a}} \mathrm{N}=429, \mathrm{~F}(2,426)=69.60, p<0.0005, R^{2}=0.25$.

${ }^{\mathrm{b}} \mathrm{N}=128, \mathrm{~F}(1,126)=0.85, p=0.36, R^{2}=0.01$.

${ }^{\mathrm{c}} \mathrm{N}=301, \mathrm{~F}(1,299)=0.49, p=0.48, R^{2}=0.00$.

${ }^{\mathrm{d}}$ Residence: urban $=1$, rural $=0$.

e Husband's occupation: white-collar $=1$, blue-collar $=0$.

$* * * * p<0.0005$.

Table 1c

Multiple Regression Analysis on Monthly Income According to Husband's Occupation: Shanghai Province

\begin{tabular}{|c|c|c|c|c|c|c|}
\hline \multirow[b]{2}{*}{ Intercept } & \multicolumn{2}{|c|}{ Total $^{\mathrm{a}}$} & \multicolumn{2}{|c|}{ Urban $^{b}$} & \multicolumn{2}{|c|}{ Rural $^{\mathrm{C}}$} \\
\hline & $\begin{array}{c}\text { Coefficient } \\
116.74\end{array}$ & $\begin{array}{l}\text { S.E. } \\
7.72^{* * * *}\end{array}$ & $\begin{array}{c}\text { Coefficient } \\
169.22\end{array}$ & $\begin{array}{l}\text { S.E. } \\
7.91^{* * * *}\end{array}$ & $\begin{array}{l}\text { Coefficient } \\
119.13\end{array}$ & $\begin{array}{c}\text { S.E. } \\
8.41^{* * * *}\end{array}$ \\
\hline $\begin{array}{l}\text { Urban versus } \\
\text { Rural }^{\text {d }}\end{array}$ & 54.73 & $10.04^{* * * *}$ & & & & \\
\hline $\begin{array}{l}\text { White- versus } \\
\text { Blue-collar }\end{array}$ & 12.81 & 10.52 & 18.16 & 12.19 & -1.36 & 20.48 \\
\hline
\end{tabular}

${ }^{\mathrm{a}} \mathrm{N}=464, \mathrm{~F}(2,461)=18.69, p<0.0005, R^{2}=0.08$.

${ }^{\mathrm{b}} \mathrm{N}=280, \mathrm{~F}(1,278)=2.22, p=0.14, R^{2}=0.01$.

${ }^{\mathrm{c}} \mathrm{N}=184, \mathrm{~F}(1,182)=0, p=0.95, R^{2}=0.00$.

${ }^{\mathrm{d}}$ Residence: urban $=1$, rural $=0$.

'Husband's occupation: white-collar $=1$, blue-collar $=0$.

${ }^{* * * *} p<0.0005$

\section{Education}

Across provinces, children in urban areas had more education than children in rural areas. The older the children were, of course, the more education they had. When all children were considered, children born to white-collar families received more schooling than those born to bluecollar families (Tables 3a-c). In the urban part of each province, children 
TIH-FEN TING

of white-collar background stayed in school longer than their blue-collar counterparts. The same pattern also holds true in the rural part of each province (Tables 3a-c).

Table 2a

Multiple Regression Analysis on Number of Living Children for Married Couples According to Husband's Occupation: Hebei Province

\begin{tabular}{|c|c|c|c|c|c|c|}
\hline \multirow[b]{2}{*}{ Intercept } & \multicolumn{2}{|c|}{ Total $^{\mathrm{a}}$} & \multicolumn{2}{|c|}{ Urban $^{\mathrm{b}}$} & \multicolumn{2}{|c|}{ Rural $^{\mathrm{C}}$} \\
\hline & $\begin{array}{c}\text { Coefficient } \\
4.23\end{array}$ & $\begin{array}{c}\text { S.E. } \\
0.08^{* * * *}\end{array}$ & $\begin{array}{c}\text { Coefficient } \\
4.19\end{array}$ & $\begin{array}{c}\text { S.E. } \\
0.13^{* * * *}\end{array}$ & $\begin{array}{c}\text { Coefficient } \\
4.18\end{array}$ & $\begin{array}{c}\text { S.E. } \\
0.08^{* * * *}\end{array}$ \\
\hline $\begin{array}{l}\text { Urban versus } \\
\text { Rural }^{\text {d }}\end{array}$ & -0.21 & 0.15 & & & & \\
\hline $\begin{array}{l}\text { White- versus } \\
\text { Blue-collar }^{\mathrm{e}}\end{array}$ & -0.59 & $0.18^{* * *}$ & -1.22 & $0.25^{* * * *}$ & -0.10 & 0.24 \\
\hline
\end{tabular}

${ }^{\mathrm{a}} N=524, \mathrm{~F}(2,521)=7.98, p<0.0005, R^{2}=0.03$.

${ }^{\mathrm{b}} \mathrm{N}=138, \mathrm{~F}(1,136)=23.87, p<0.0005, R^{2}=0.15$.

${ }^{\mathrm{c}} N=386, \mathrm{~F}(1,384)=0.18, p=0.68, R^{2}=0.00$.

${ }^{\mathrm{d}}$ Residence: urban $=1$, reural $=0$.

eHusband's occupation: white-collar $=1$, blue-collar $=0$

$* * * p<0.005$.

$* * * * p<0.0005$

Table 2b

Multiple Regression Analysis on Number of Living Children for Married Couples According to Husband's Occupation: Shaanxi Province

\begin{tabular}{|c|c|c|c|c|c|c|}
\hline \multirow[b]{2}{*}{ Intercept } & \multicolumn{2}{|c|}{ Total $^{\mathrm{a}}$} & \multicolumn{2}{|c|}{ Urban $^{\mathrm{b}}$} & \multicolumn{2}{|c|}{ Rural $^{\mathrm{C}}$} \\
\hline & $\begin{array}{c}\text { Coefficient } \\
4.52\end{array}$ & $\begin{array}{c}\text { S.E. } \\
0.09^{* * * *}\end{array}$ & $\begin{array}{c}\text { Coefficient } \\
4.07\end{array}$ & $\begin{array}{c}\text { S.E. } \\
0.14^{* * * *}\end{array}$ & $\begin{array}{c}\text { Coefficient. } \\
4.49\end{array}$ & $\begin{array}{c}\text { S.E. } \\
0.10^{* * * *}\end{array}$ \\
\hline $\begin{array}{l}\text { Urban versus } \\
\text { Rural }^{\text {d }}\end{array}$ & -0.52 & $0.17^{* * *}$ & & & & \\
\hline $\begin{array}{l}\text { White- versus } \\
\text { Blue-collare }\end{array}$ & -0.39 & 0.22 & -0.62 & $0.25^{*}$ & -0.10 & 0.35 \\
\hline
\end{tabular}

${ }^{\mathrm{a}} \mathrm{N}=429, \mathrm{~F}(2,426)=8.83, p<0.0005, R^{2}=0.04$.

${ }^{\mathrm{b}} \mathrm{N}=128, \mathrm{~F}(1,126)=6.26, p<0.05, R^{2}=0.05$.

${ }^{\mathrm{c}} N=301, \mathrm{~F}(1,299)=0.09, p=0.77, R^{2}=0.00$.

${ }^{\mathrm{d}}$ Residence: urban $=1$, rural $=0$.

eHusband's occupation: white-collar $=1$, blue-collar $=0$.

$* p<0.5$.

${ }^{* * *} p<0.005$

**** $p<0.0005$ 
Table 2c

Multiple Regression Analysis on Number of Living Children for Married Couples According to Husband's Occupation: Shanghai Province

\begin{tabular}{|c|c|c|c|c|c|c|}
\hline \multirow[b]{2}{*}{ Intercept } & \multicolumn{2}{|c|}{ Total $^{\mathrm{a}}$} & \multicolumn{2}{|c|}{ Urban $^{\mathrm{b}}$} & \multicolumn{2}{|c|}{ Rural $^{\mathrm{C}}$} \\
\hline & $\begin{array}{c}\text { Coefficient } \\
2.88\end{array}$ & $\begin{array}{c}\text { S.E. } \\
0.07^{* * * *}\end{array}$ & $\begin{array}{c}\text { Coefficient } \\
2.65\end{array}$ & $\begin{array}{c}\text { S.E. } \\
0.07^{* * * *}\end{array}$ & $\begin{array}{c}\text { Coefficient } \\
2.80\end{array}$ & $\begin{array}{c}\text { S.E. } \\
0.08^{* * * *}\end{array}$ \\
\hline $\begin{array}{l}\text { Urban versus } \\
\text { Rural }^{\mathrm{d}}\end{array}$ & -0.29 & $0.09 * * *$ & & & & \\
\hline $\begin{array}{l}\text { White- versus } \\
\text { Blue-collare }\end{array}$ & -0.37 & $0.10^{* * * *}$ & -0.54 & $0.11 * * * *$ & 0.07 & 0.19 \\
\hline
\end{tabular}

${ }^{\mathrm{a}} \mathrm{N}=464, \mathrm{~F}(2,461)=16.37, p<0.0005, R^{2}=0.07$.

${ }^{\mathrm{b}} \mathrm{N}=280, \mathrm{~F}(1,278)=22.60, p<0.0005, R^{2}=0.08$.

${ }^{\mathrm{c}} \mathrm{N}=184, \mathrm{~F}(1,182)=0.13, p=0.72, R^{2}=0.00$.

${ }^{\mathrm{d}}$ Residence: urban $=1$, rural $=0$.

${ }^{\mathrm{e}}$ Husband's occupation: white-collar $=1$, blue-collar $=0$.

$* * * p<0.005$

$* * * * p<0.0005$

Table 3a

Multiple Regression Analysis on Years of Education Children Received According to Father's Occupation: Hebei Province

\begin{tabular}{|c|c|c|c|c|c|c|}
\hline \multirow[b]{2}{*}{ Intercept } & \multicolumn{2}{|c|}{ Total $^{\mathrm{a}}$} & \multicolumn{2}{|c|}{$U_{\text {rban }}^{b}$} & \multicolumn{2}{|c|}{ Rural $^{\mathrm{C}}$} \\
\hline & $\begin{array}{c}\text { Coefficient } \\
0.16\end{array}$ & $\begin{array}{l}\text { S.E. } \\
0.30\end{array}$ & $\begin{array}{c}\text { Coefficient } \\
0.89\end{array}$ & $\begin{array}{l}\text { S.E. } \\
0.63\end{array}$ & $\begin{array}{c}\text { Coefficient } \\
0.32\end{array}$ & $\begin{array}{l}\text { S.E. } \\
0.34\end{array}$ \\
\hline $\begin{array}{l}\text { Urban versus } \\
\text { Rural }^{\mathrm{d}}\end{array}$ & 1.14 & $0.17^{* * * *}$ & & & & \\
\hline Child's Age & 0.34 & $0.02^{* * * *}$ & 0.37 & $0.03^{* * * *}$ & 0.32 & $0.02 * * * *$ \\
\hline $\begin{array}{l}\text { Male versus } \\
\text { Female }\end{array}$ & 0.48 & $0.14^{* * *}$ & -0.16 & 0.29 & 0.67 & $0.16^{* * * *}$ \\
\hline $\begin{array}{l}\text { White- versus } \\
\text { Blue-collar }^{f}\end{array}$ & 1.47 & $0.22^{* * * *}$ & 2.02 & $0.35^{* * * *}$ & 1.09 & $0.27^{* * * *}$ \\
\hline
\end{tabular}

${ }^{\mathrm{a}} \mathrm{N}=1525, \mathrm{~F}(4,1520)=138.68, p<0.0005, R^{2}=0.27$.

${ }^{\mathrm{b}} \mathrm{N}=345, \mathrm{~F}(3,341)=54.39, p<0.0005, R^{2}=0.32$.

${ }^{\mathrm{c}} \mathrm{N}=1180, \mathrm{~F}(3,1176)=109.61, p<0.0005, R^{2}=0.22$.

${ }^{\mathrm{d}}$ Residence: urban $=1$, rural $=0$.

${ }^{\mathrm{e} C h i l d}$ 's sex: male $=1$, female $=0$.

${ }^{f}$ Father's occupation: white-collar $=1$, blue-collar $=0$.

${ }^{* * *} p<0.005$

$* * * * p<0.0005$ 
TIH-FEN TING

Table $3 \mathbf{b}$

\section{Multiple Regression Analysis on Years of Education Children Received According to Father's Occupation: Shaanxi Province}

\begin{tabular}{|c|c|c|c|c|c|c|}
\hline \multirow[b]{2}{*}{ Intercept } & \multicolumn{2}{|c|}{ Total $^{\mathrm{a}}$} & \multicolumn{2}{|c|}{$U_{r b a n}^{b}$} & \multicolumn{2}{|c|}{ Rural $^{\mathrm{C}}$} \\
\hline & $\begin{array}{l}\text { Coefficient } \\
-0.84\end{array}$ & $\begin{array}{c}\text { S.E. } \\
0.28^{* * *}\end{array}$ & $\begin{array}{c}\text { Coefficient } \\
1.43\end{array}$ & $\begin{array}{c}\text { S.E. } \\
0.50 * * *\end{array}$ & $\begin{array}{c}\text { Coefficient } \\
-0.72\end{array}$ & $\begin{array}{r}\text { S.E. } \\
0.33^{*}\end{array}$ \\
\hline $\begin{array}{l}\text { Urban versus } \\
\text { Rural }^{\text {d }}\end{array}$ & 2.68 & $0.18^{* * * *}$ & & & & \\
\hline Child's Age & 0.38 & $0.02 * * * *$ & 0.42 & $0.03 * * * *$ & 0.36 & $0.02 * * * *$ \\
\hline $\begin{array}{l}\text { Male versus } \\
\text { Female }^{\mathrm{e}}\end{array}$ & 0.09 & 0.15 & -0.44 & 0.24 & 0.29 & 0.19 \\
\hline $\begin{array}{l}\text { White- versus } \\
\text { Blue-collar }^{f}\end{array}$ & 0.97 & $0.22^{* * * *}$ & 0.53 & $0.26^{*}$ & 1.35 & $0.33^{* * * *}$ \\
\hline
\end{tabular}

${ }^{\mathrm{a}} N=1250, \mathrm{~F}(4,1245)=259.23, p<0.0005, R^{2}=0.45$.

${ }^{\mathrm{b}} N=318, \mathrm{~F}(3,314)=88.85, p<0.0005, R^{2}=0.46$.

${ }^{\mathrm{c}} \mathrm{N}=932, \mathrm{~F}(3,928)=145.40, p<0.0005, R^{2}=0.32$.

${ }^{\mathrm{d}}$ Residence: urban $=1$, rural $=0$.

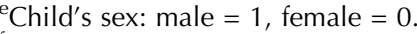

fFather's occupation: white-collar $=1$, blue-collar $=0$.

${ }^{*} p<0.05$.

$* * * p<0.005$

$* * * * p<0.0005$.

Thus, urban couples had fewer children than rural couples, but their children received more years of schooling than children of rural couples. Within each province, urban white-collar couples also had fewer children than urban blue-collar couples, but their children also received more schooling than children of urban blue-collar couples. Although no difference in lifetime reproductive success was found between white-collar and blue-collar couples in rural areas, children of white-collar background had significantly more education than children of blue-collar background.

\section{DISCUSSION}

The women in this study (aged 45-49 in 1985), along with their spouses, went through their prime reproductive years during the first 35 years of history of the People's Republic of China. Many of the political events, and state policies, of that period are the sorts that alter reproductive costs and benefits for couples of different occupational backgrounds. For example, the central government embarked on several campaigns (e.g., the anti- 
Table 3c

Multiple Regression Analysis on Years of Education Children Received According to Father's Occupation: Shanghai Province

\begin{tabular}{|c|c|c|c|c|c|c|}
\hline \multirow[b]{2}{*}{ Intercept } & \multicolumn{2}{|c|}{ Total $^{\mathrm{a}}$} & \multicolumn{2}{|c|}{ Urban $^{b}$} & \multicolumn{2}{|c|}{ Rural $^{\mathrm{C}}$} \\
\hline & $\begin{array}{c}\text { Coefficient } \\
4.28\end{array}$ & $\begin{array}{c}\text { S.E. } \\
0.40^{* * * *}\end{array}$ & $\begin{array}{c}\text { Coefficient } \\
6.86\end{array}$ & $\begin{array}{c}\text { S.E. } \\
0.50^{* * * *}\end{array}$ & $\begin{array}{c}\text { Coefficient } \\
3.24\end{array}$ & $\begin{array}{c}\text { S.E. } \\
0.73^{* * * * *}\end{array}$ \\
\hline $\begin{array}{l}\text { Urban versus } \\
\text { Rural }^{\text {d }}\end{array}$ & 1.99 & $0.15^{* * * *}$ & & & & \\
\hline Child's Age & 0.20 & $0.02 * * * *$ & 0.18 & $0.02 * * * *$ & 0.25 & $0.04 * * * *$ \\
\hline $\begin{array}{l}\text { Male versus } \\
\text { Female }\end{array}$ & -0.02 & 0.14 & -0.18 & 0.17 & 0.23 & 0.24 \\
\hline $\begin{array}{l}\text { White- versus } \\
\text { Blue-collar }^{f}\end{array}$ & 0.90 & $0.16^{* * * *}$ & 0.80 & $0.18^{* * * *}$ & 1.09 & $0.32^{* * *}$ \\
\hline
\end{tabular}

${ }^{\mathrm{a}} \mathrm{N}=906, \mathrm{~F}(4,901)=110.41, p<0.0005, R^{2}=0.33$.

${ }^{\mathrm{b}} \mathrm{N}=558, \mathrm{~F}(3,554)=25.08, p<0.0005, R^{2}=0.12$.

${ }^{\mathrm{c}} \mathrm{N}=348, \mathrm{~F}(3,344)=18.66, p<0.0005, R^{2}=0.14$.

${ }^{\mathrm{d}}$ Residence: urban $=1$, rural $=0$.

e Child's sex: male $=1$, female $=0$.

${ }^{\mathrm{f}}$ Father's occupation: white-collar $=1$, blue-collar $=0$.

*** $p<0.005$

$* * * * p<0.0005$

rightist campaign in the late 1950s, and the Cultural Revolution of the 1960s and 1970s) to downgrade the status of intellectuals and upgrade the status of manual workers (Lin \& Xie, 1988; Schoppa, 2002). In an effort to eliminate capitalist societies' privileging of white-collar professions over blue-collar jobs, much income differentiation between white-collar and blue-collar workers was eliminated or at least compressed, especially during the period of the Cultural Revolution (Lin \& Xie, 1988; Parish, 1984). At the same time, for educational opportunities, the children of peasants and workers were favored-at least, in policy_over children from white-collar families (Deng \& Treiman, 1997; Parish, 1984; Zhou, Moen \& Tuma, 1998).

Despite state interventions reducing class differences during revolutionary China, in my study a clear pattern emerges in all provinces: whitecollar couples had a different parental investment strategy than blue collar couples. Specifically, urban white-collar couples had fewer but bettereducated children (i.e., more years of schooling) than their blue-collar counterparts; rural white-collar couples also had better educated children even though they had as many children as rural blue-collar couples. In both urban and rural areas, couples of different occupational backgrounds diverged in their parental investment strategies. 
TIH-FEN TING

Although I found no differences in monthly income between whitecollar and blue-collar couples within either urban or rural areas, across provinces rural couples consistently had lower monthly income than urban couples. Rural children also consistently had fewer years of schooling than urban children. Comparatively, low population density and unskilled labor market (farming-related work accounts for the majority of labor) in rural areas do not create the same kind of competitive environment as in urban areas which have high population densities. Moreover, the government policy of restricted mobility between urban and rural residence also made it nearly impossible for rural residents to live and work in the cities, except for attending higher education institutions and joining the army (Stockman, 2000). Once rural children reached 16 (minimum working age), they automatically entered the labor force of their production teams and started to earn income under agricultural collectivization (Peng, 1991, p.79).

Thus, increased parental investment in offspring quality is not necessary to ensure a child's ability to secure a job in rural China (cf. Kaplan \& Lancaster, 2000). Nevertheless, in rural areas, white-collar parents with embodied cultural capital, such as knowledge and access to resources, provided their children with more education than children of blue-collar background - with no reduction in the number of children.

The fertility transition took place in China's urban areas long before it happened in rural areas (Peng, 1991; Scharping, 2003). Here, I found that urban environments are where trade-offs between offspring quantity and quality follow couples' occupational backgrounds. The quantity-quality trade-off has been central to both economic and evolutionary approaches in explaining fertility transition (Becker, 1991; Becker \& Lewis, 1973; Borgerhoff Mulder, 2000; Kaplan, 1996; Kaplan \& Lancaster, 2000; Lam, 2003; Low, 2000b; Low et al., 1992;MacArthur \& Wilson, 1967; Willis, 1973).

The common empirical observation of the fertility transition is that higher-income and better-educated couples have fewer children than lower-income and less educated couples. A decrease in the number of children is offset by an increase in the resources invested in each child, such as resources for education (see Lam, 2003). Thus, in modern societies with low child and adult mortality, the payoffs to parental investment in offspring quality are likely to be large when wages are determined by skill and formal education (see Kaplan \& Lancaster, 2000).

However, income, wages, and educational cost cannot fully explain the trade-offs between offspring quantity and quality among couples of different occupational backgrounds in urban China. During the first three decades of the People's Republic of China, a wage structure determined by 
POPULATION AND ENVIRONMENT

the state was not correlated with skill and educational qualifications. The state, in fact, meant to eliminate income differences among different social classes.

As a result, formal education was not an important determinant of personal income (Stockman, 2000). Indeed, I found no differences in the monthly incomes of white-collar and blue-collar couples in my study. Further, educational cost was minimum because, to equalize educational opportunities, the communist government not only expanded the educational system but also created affordable primary and secondary education and free tertiary education (Deng \& Treiman, 1997). Therefore, blue-collar children presumably were just as likely as white-collar children to go to school at any level.

In short, before the economic reform, two features of communist China deviate from the preconditions of the competitive labor market model (e.g., Kaplan \& Lancaster, 2000). First, there was no or little income differentiation (except the differences between urban and rural areas). Second, there was no or little correlation between education and income. Thus, we must ask: what mechanism would then account for the observed trade-offs between offspring quantity and quality among urban couples in China? Education might be affordable to everyone but, in order to advance to the levels beyond primary schools, students had to pass the entrance examination at every level; only during the Cultural Revolution, when advancement was solely based on class origin and political recommendation, were all examinations abolished (Parish, 1984; Wang, 2000). Typically, the higher the level, the stronger was the competition.

White-collar couples, in this sample as well as in general, were bettereducated than blue-collar couples, and thus presumably able to help their children with school and educational advancement more efficiently than their less-educated blue-collar counterparts (Kaplan, 1994). In economic terms, if white-collar parents were able to lower the amount of time required to produce a unit of quality in their children (i.e., reducing the shadow price of children), then they would reach diminishing returns on investments in children at higher levels than blue-collar parents (Becker, 1991; Kaplan \& \& Lancaster, 2000; Lam, 2003). As a result, the required investment may have limited the number of children white-collar parents could afford (see also Low, 2000b; Low et al., 1992).

Increased parental investments should return large payoffs for whitecollar parents in urban areas, even though they had fewer children than blue-collar parents. Despite movements and policies specifically aimed at revamping social stratification during the first three decades of the communist regime, surveys carried out in urban cities during the 1980s revealed 
that, across gender, age, and occupational groups, professionals (e.g., physicians, scientists, engineers, writers, teachers, and officials) were still rated as the most prestigious occupations, while heavy-labor workers and service workers were among the least prestigious occupations (Bian, 1996; Lin \& Xie, 1988).

More important than income, members from higher-ranking occupations were able to take advantage of better collective goods offered by their work units. Such collective goods included better housing, health care, child care, pension, recreation, and high-quality schooling that were neither provided universally by the state nor available on the market (Stockman, 2000). Taken all together, members from higher-ranking work units, such as white-collar professionals, had a better quality of life than blue-collar workers. At the same time, urban jobs were assigned by the state on the basis of academic merit and recommendation from one's school; higher levels of education were also required for white-collar professions (Parish, 1984).

Further, during times of economic constriction, children of blue-collar backgrounds were the first to experience adversities. For example, increasing urban unemployment, beginning in the early 1960s right after the Great Leap Forward, forced the government to send youth to the countryside in hopes of alleviating growing unemployment problems in urban China. Often, worker and peasant children with poor academic performance were sent first (Parish, 1984). Academic achievement thus seemed to exert significant impacts on the life course of the youth. It was perhaps no accident that white-collar professions were at the top of the prestige scale.

Regardless of state policies toward interrupting the intergenerational inheritance of cultural capital, parents' education and occupation still affected the educational and occupational attainment for their children significantly. For example, Zhou, Moen, and Tuma (1998) report that the proportions advancing to both senior high school and college level were higher for those whose fathers were cadres or professionals than for those whose fathers were workers or peasants between 1949 and 1994; these differences were drastically reduced during the Cultural Revolution. In fact, for the first time, the proportion of worker and peasant children in college exceeded that of white-collar children briefly during the mid-1970s. In other words, fathers with more education and higher positions produced children who got further in the educational system; those children, in turn, tended to get better jobs than others (Parish, 1984).

Thus, the reproduction, even inheritance, of social status and cultural capital continued until the Cultural Revolution, when it was effectively broken down. Although, the Cultural Revolution succeeded in reducing 
the intergenerational transmission of cultural and social capital in the short run, intergenerational educational correlations immediately made a comeback as soon as the era ended (Deng \& Treiman, 1997; Zhou et al., 1998).

In contrast with rural areas in China, high population density and an occupational structure that incidentally helped reinforce unequal distribution of cultural capital in the population encouraged urban couples with different levels of resources (i.e., cultural capital rather than income in this case) to adopt different reproductive strategies. To be sure, Mao's China was much more egalitarian than both industrial capitalist, and less developed, countries from the same period (Parish, 1984). Nonetheless, we still observe the trade-offs between offspring quality and quantity for urban couples with different occupations, which suggests how sensitive human parental investment strategies can be.

\section{REFERENCES}

Anderson, K. G., Kaplan, H., \& Lancaster, J. B. (1999). Parental care by genetic fathers and stepfathers I: Reports from Albuquerque man. Evolution and Human Behavior 20, 405431.

Becker, G. S. (1991). A Treatise on the Family. Cambridge, MA: Harvard University Press.

Becker, G. S., \& Lewis, H. G. (1973). Interaction between quantity and quality of children. In T. W. Schultz (Ed.), Economics of the Family: Marriage, Children, and Human Capital (pp. 81-90). Chicago, IL: University of Chicago Press.

Bian, Y. (1996). Chinese occupational prestige: A comparative analysis. International Sociology 11, 161-186.

Borgerhoff Mulder, M. (2000). Optimizing offspring: The quantity-quality tradeoff in agropastoral Kipsigis. Evolution and Human Behavior 21, 391-410.

Charnov, E. L. (1982). The Theory of Sex Allocation. NJ: Princeton University Press.

Clutton-Brock, T., \& Godfray, C. (1991). Parental investment. In J. R. Krebs, \& N. B. Davis (Eds.), Behavioural Ecology: An Evolutionary Approach (pp. 32-68). London: Blackwell.

Dankert, G., \& van Ginneken, J. (1991). Birth weight and other determinants of infant and child mortality in three provinces of China. Journal of Biosocial Science 23, 477-489.

Deng, Z., \& Treiman, D. J. (1997). The impact of the Cultural Revolution on the trends in educational attainment in the People's Republic of China. American Journal of Sociology 103, 391-428.

Goody, J. (1983). The Development of the Family and Marriage in Europe. Cambridge University Press.

Hannum, E., \& Xie, Y. (1994). Trends in educational gender inequality in China: 1949-1985. Social Stratification and Mobility 13, 73-98.

Kaplan, H. (1994). Evolutionary and wealth flows theories of fertility: Empirical tests and new models. Population and Development Review 20, 753-791.

Kaplan, H. (1996). A theory of fertility and parental investment in traditional and modern human societies. Yearbook of Physical Anthropology 39, 91-135.

Kaplan, H., \& Lancaster, J. B. (2000). The evolutionary economics and psychology of the demographic transition to low fertility. In L. Cronk, N. Chagnon, \& W. Irons (Eds.), Adaptation and Human Behavior: An Anthropological Perspective (pp. 283-322). New York: Aldine de Gruyter. 
TIH-FEN TING

Lam, D. (2003). Evolutionary biology and rational choice in models of fertility. In K. W. Wachter, \& R. A. Bulatao (Eds.), Offspring: Human Fertility Behavior in Biodemographic Perspective (pp. 322-338). Washington, DC: National Academies Press.

Lancaster, J. B. (1997). The evolutionary history of human parental investment in relation to population growth and social stratification. In P. A. Gowaty (Ed.), Feminism and Evolutionary Biology (pp. 466-488). New York: Chapman Hall.

Lavely, W., Xiao, Z., Li, B., \& Freedman, R. (1990). The rise in female education in China: National and regional patterns. China Quarterly 121, 61-93.

Lessells, C. M. (1991). The evolution of life history. In J. R. Krebs \& N. B. Davis (Eds.), Behavioural Ecology: An Evolutionary Approach (pp. 32-68). London: Blackwell.

Lin, N., \& Xie, W. (1988). Occupational prestige in urban China. American Journal of Sociology $93,793-832$

Low, B. S. (2000a). Why Sex Matters: A Darwinian Look at Human Behavior. NJ: Princeton University Press.

Low, B. S. (2000b). Sex, wealth, and fertility: Old rules, new environments. In L. Cronk, N. Chagnon, \& W. Irons (Eds.), Adaptation and Human Behavior: An Anthropological Perspective (pp. 323-344). New York: Aldine de Gruyter.

Low, B. S., Clarke, A. L., and Lockridge, K. A. (1992). Toward an ecological demography. Population and Development Review 18, 1-31.

MacArthur, R. H., \& Wilson, E. O. (1967). The Theory of Island Biogeography. NJ: Princeton University Press.

Meisner, M. (1999). Mao's China and After: A History of the People's Republic. 3rd ed. New York: The Free Press.

Parish, W. (1984). Destratification in China. In J. Watson (Ed.), Class and Social Stratification in Post-Revolution China (pp. 84-120). MA: Cambridge University Press.

Peng, X. (1991). Demographic Transition in China: Fertility Trends since the 1950s. Oxford: Clarendon Press.

Roff, D. A. (1992). The Evolution of Life Histories: Theory and Analysis. New York: Chapman and Hall.

Scharping, T. (2003). Birth Control in China 1949-2000. London: Routeledge Curzon.

Schoppa, R. K. (2002). Revolution and its Past: Identities and Change in Modern Chinese History. NJ: Prentice Hall.

State Statistical Bureau (1986). China In-Depth Fertility Survey. Principal Report, Vols $1 \& 2$. Department of Population Statistics of the State Statistical Bureau, Beijing.

Stearns, S. C. (1992). The Evolution of Life History. Oxford University Press.

Stockman, N. (2000). Understanding Chinese Society. Cambridge: Polity Press.

Ting, T. -F. 2003. Human reproductive ecology in revolutionary China. PhD Dissertation. University of Michigan.

Wang, G. (2000). The distribution of China's population and its changes. In X. Peng \& Z. Guo (Eds.), The Changing Population of China (pp. 11-19). London: Blackwell.

Willis, R. J. (1973). A new approach to the economic theory of fertility behavior. Journal of Political Economy (March-April), S14-S64.

Zhou, X., Moen, P., \& Tuma, N. B. (1998). Educational stratification in urban China: 1949-94. Sociology of Education 71, 199-222. 